Vol. 53: 1, 2008

doi: 10.3354/ame01219
AQUATIC MICROBIAL ECOLOGY

Aquat Microb Ecol
Printed September 2008

Published online September 18, 2008

Contribution to AME Special 1 'Progress and perspectives in aquatic microbial ecology'

\title{
INTRODUCTION
}

\section{Progress, challenges, and perspectives in marine microbial ecology}

\author{
Paul A. del Giorgio* \\ Département des sciences biologiques, Université du Québec à Montréal (UQÀM), CP 8888, succursale Centre Ville, \\ Montréal, Québec H3C 3P8, Canada
}

When observed from above, the surfaces of oceans, estuaries and littoral areas show beautiful and complex patterns and textures. Some of these large-scale surface features are visible from space, and many are in fact generated by the smallest oceanic inhabitants, prokaryotic and eukaryotic unicellular organisms. A similar heterogeneity exists along a depth axis, and a vertical oceanic tour would reveal not only intricate and dynamic physical structures, but also complex biological patterns, particularly at the microbial scale. One of the main challenges of marine microbial ecology has traditionally been to connect these disparate spatial scales that coexist in our aquatic biosphere, and to extrapolate the microbial features and processes typically measured at very small spatial and temporal scales to scales that are relevant to the functioning of oceanic systems. There are logistic, technical and conceptual difficulties associated with this scaling; some of these challenges, such as the spatial and temporal distribution of bacterial production and growth in the oceans, have been the research focus of our community for decades now, and yet we still have much to learn; some challenges are more recent, for example the exploration of spatial and temporal patterns of marine microbial diversity. New paradigms and challenges are constantly emerging, such as the distribution and biogeochemical importance of novel microbial metabolic pathways in the ocean.
Many of these existing and emerging challenges and research opportunities in our discipline were presented and discussed during the 10th Symposium on Aquatic Microbial Ecology (SAME 10), held in Faro (Portugal) in September 2007, through a combination of invited plenary talks, targeted thematic workshops and individual contribution of participants. This first special AME issue brings together the highlights of this meeting and presents some of the main topics that were explored. AME Special 1 provides a synthesis of a diverse array of key current topics in marine microbial ecology, including new developments in the measurement of microbial community structure and processes in biofilms, sediments, and the water column, patterns of microbial diversity and their ecological and biogeochemical significance, the current state of our understanding of major oceanic biogeochemical processes, of key biological interactions such as predation, and of specific microbial components, as well as conceptual issues concerning microbial services at the global scale. It is clear that we have a long way to go and that there will be no lack of future challenges, surprises and difficulties, but our community has made great progress in the past decade, and this special AME issue is a reflection of these achievements. I thank all the authors who so effectively contributed to develop this project. 